Journal of Data Science 829-856 , DOI: 10.6339/JDS.201810_16(4).00009

\title{
AN INTERVAL-CENSORED PROPORTIONAL HAZARDS MODEL
}

\author{
John M. Williamson ${ }^{*}$, Hung-Mo Lin ${ }^{1}$, Hae-Young Kim² \\ *Division of Parasitic Diseases and Malaria, National Center of Global Health, \\ Centers for Disease Control and Prevention (MS A-06), 1600 Clifton Road, Atlanta, GA \\ 30329, U.S.A. \\ ${ }^{1}$ Department of Population Health Science and Policy, Icahn School of Medicine at \\ Mount Sinai, One Gustave L. Levy Place, Box 1077, New York, NY 10029, U.S.A. \\ ${ }^{2}$ Department of Public Health, New York Medical College, 40 Sunshine Cottage Rd, \\ Valhalla, NY 10595, U.S.A.
}

\begin{abstract}
We fit a Cox proportional hazards (PH) model to interval-censored survival data by first subdividing each individual's failure interval into nonoverlapping sub-intervals. Using the set of all interval endpoints in the data set, those that fall into the individual's interval are then used as the cut points for the sub-intervals. Each sub-interval has an accompanying weight calculated from a parametric Weibull model based on the current parameter estimates. A weighted PH model is then fit with multiple lines of observations corresponding to the sub-intervals for each individual, where the lower end of each sub-interval is used as the observed failure time with the accompanying weights incorporated. Right-censored observations are handled in the usual manner. We iterate between estimating the baseline Weibull distribution and fitting the weighted $\mathrm{PH}$ model until the regression parameters of interest converge. The regression parameter estimates are fixed as an offset when we update the estimates of the Weibull distribution and recalculate the weights. Our approach is similar to Satten et al.'s (1998) method for interval-censored survival analysis that used imputed failure times generated from a parametric model in a PH model. Simulation results demonstrate apparently unbiased parameter estimation for the correctly specified Weibull model and little to no bias for a mis-specified log-logistic
\end{abstract}

${ }^{*}$ Corresponding author

Email: jow5@cdc.gov 
model. Breast cosmetic deterioration data and ICU hyperlactemia data are analyzed.

Key words: Accelerated failure time model; Interval-censored failure time data; Parametric survival analysis; Proportional hazards model 


\section{Introduction}

Interval-censored failure data are a special case of survival data in which the only information available to the investigator is whether an event occurred before or after one or more visit (examination) times. For example, in HIV studies, investigators cannot observe the exact moment when the virus develops, only whether the virus developed before or after the test. Interval-censored failure data often occur in observational or follow-up studies where patients are not continuously being observed. Whether or not the event occurred is ascertained at the observation times, and the failure time of the event itself is not available. Such data are increasing in medical studies due in part to the greater use of biomarkers that define a disease progression endpoint (Heller (2011)). A special case of interval-censored data is current-status data, where individuals are seen only once after enrollment (Diamond et al. (1986), Grummer-Strawn (1993)). Thus, the observations are either of the form $(0, \mathrm{C}]$ or $(\mathrm{C}, \infty)$ (i.e., left- or right- censored). These data are also commonly referred to as case 1 interval-censored data (Huang (1996)).

There have been numerous methods proposed for the analysis of interval-censored failure data. Peto and Peto (1972) first considered the comparison of the intervalcensored survival curves of two samples. Finkelstein (1986) proposed a semiparametric method in which the baseline distribution and regression parameters are fit simultaneously by maximizing the full likelihood of the data. Sun (1996) proposed a test statistic for interval-censored failure data having the same algebraic form as the original log-rank test. Zhao and Sun (2004) generalized Sun's log-rank test (1996) to include exact failure times in interval-censored data. Sun, Zhao, and Zhao (2005) proposed a class of non-parametric tests for the comparison of $\mathrm{k}$ interval-censored survival curves that are generalizations of Peto and Peto's log-rank test (1972).

Satten (1996) considered a marginal likelihood approach to fitting the proportional hazards (PH) model (Cox (1972), Cox (1975)) by maximizing a likelihood that is the sum over all rankings of the data that are consistent with the observed censoring intervals. Satten et al. (1998) suggested a parametric model for the baseline hazard to generate imputed failure times. In their model the usual PH model for right-censored data is used to estimate the regression parameters. Heller (2011) proposed a method for estimation and inference of the regression parameters in the Cox PH model with interval censoring based on estimating equations and using an inverse probability weight to select event time pairs where the ordering is unambiguous. A Bayesian estimation approach has recently been proposed for analyzing interval-censored data under the PH model (Lin et al. (2015)). The PH models and tests referenced above for analyzing interval-censored data can be used for the analysis of current status data. Murphy and van der Vaart (1997) considered semiparametric likelihood ratio inference 
and proposed a test for significance of the regression coefficient in Cox's regression model for current status data.

To fit a Cox PH model to interval-censored failure time data, we begin by subdividing each individual's failure interval into non-overlapping sub-intervals. Using the set of all interval endpoints in the data set, those that fall into the individual's interval are then used as the cut points for the sub-intervals. Then for each sub-interval, an exact failure time is assumed and the accompanying weight is calculated. The sum of the weights for each individual is 1.0. Our approach is similar to Satten et al.'s method (1998) for interval-censored survival analysis who used imputed failure times generated from a parametric model in a PH model.

We present the details of our approach in Section 2. In Section 3 we present simulation studies to detail its performance and compare it with Satten et al.'s approach (1998). We illustrate the proposed PH approach in Section 4 with analysis of breast cancer study data and intensive care unit hyperlactemia data. We conclude with a short discussion on the merits of the proposed approach.

\section{Methods}

Let $T_{i}$ denote the log-transformed failure time for the $i^{\text {th }}$ observation $(i=1, \ldots, n$ where $n$ is the sample size). If data are interval censored, then for each individual, instead of a failure time, we observe a censoring interval $\left(l_{i}, u_{i}\right]$ that is known to contain the actual failure time. The failure indicator is defined as $\delta_{i}=1$ if the $i^{\text {th }}$ observation is of the form $\left(l_{i}, u_{i}\right]$ (interval censored, or left-censored if $\left.l_{i}=0\right)$. For right-censored observations $\delta_{i}=0$. We assume throughout that the censoring/dropout mechanism is independent of both the response time and the covariates.

Let the survivor distribution for $T$ be denoted by $S(t ; \theta, \beta)=\operatorname{Pr}(T \geq t)$, where $t \geq 0, \theta$ is a column vector of intercept $(\Delta)$ and scale $(\sigma)$ parameters, and $\beta$ is a $(p \times 1)$ column vector of regression parameters. The log-likelihood for such interval-censored failure data is

$$
\mathrm{l}\left(l_{i}, u_{i}, \delta_{i}, \beta, \theta\right)=\sum_{i=1}^{n}\left\{\delta_{i} \log S\left(l_{i}\right)-S\left(u_{i}\right)+\left(1-\delta_{i}\right) \log \left[S\left(l_{i}\right)\right]\right\}
$$

with $S(0)=1$. We assume that the observed data comprise iid samples. Further assume that $T$ follows a Weibull distribution. Let $z_{i}=\left(u_{i}-\Delta-x_{i}{ }^{\prime} \beta_{A F T}\right) / \sigma$ and $v_{i}=\left(l_{i}-\right.$ $\left.\Delta-x_{i}{ }^{\prime} \beta_{A F T}\right) / \sigma$, where $x_{i}$ is a column vector of mean-centered covariates and $\beta_{A F T}$ are parameters fit with an accelerated failure time (AFT) model. Then $S\left(z_{i}\right)=$ $\exp \left\{-\exp \left(z_{i}\right)\right\}, f\left(z_{i}\right)=(1 / \sigma) \exp \left(z_{i}\right) \exp \left\{-\exp \left(z_{i}\right)\right\}$ and the corresponding survivor and pdf functions for $v_{i}$. Also assume the baseline survival function $S(t)$ at a given event time $t$ can be estimated when each component of the covariate vector $x$ equals 
zero. The Weibull distribution belongs to both the AFT and PH families. Therefore, there is an interchangeable relationship between the $\beta$ parameters fit by the parametric Weibull AFT and PH models: $\beta_{A F T}=-\sigma \times \beta_{P H}$. Hereafter, if $\beta$ has no subscript then it refers to the $\mathrm{PH}$ parameters, $\beta_{P H}$. We also choose the Weibull model for the underlying distribution because of its flexibility and easy generalizability to other settings (Alkarni (2016), Mustafa et al. (2016), Pu et al. (2016)).

Here we propose to estimate $\beta_{P H}$ based on the partial likelihood derived from the $\mathrm{PH}$ model, instead of a full likelihood approach where we would be required to make a parametric assumption on the failure time distribution. The partial likelihood only assigns a relative weighting to the possible rank orderings of failure times and is less sensitive to misspecification of the form of the failure time distribution than a fully parametric based likelihood, which requires that the distribution of $T$ be correctly specified (Satten et al. (1998)). Specifying the failure distribution can be challenging for interval-censored failure data as the exact failure times are not observed. Fitting a Cox PH model requires knowledge of the exact failure (censoring) times of individuals in the study in order to obtain the ranking of observations for computation of the partial likelihood. Satten et al. (1998) proposed to impute a number of failure times within each observed interval for interval-censored individuals. A parametric distribution for the failure time data needs to be assumed for imputation; however, the imputed data are then analyzed using the $\mathrm{PH}$ model. They propose to estimate $\beta$ solving

$$
S_{\beta}(\beta, \theta)=E_{F}[S(t \mid \delta, X, \beta)]=\int S(t \mid \delta, X ; \beta) d F(t \mid \delta, X ; \beta)
$$

where $F(t \mid \delta, X ; \beta)$ is a parametric family of conditional distributions of failure times $t$ given the observed censoring intervals, covariates, and right-censored indicator $\delta$, and assuming that this family of distributions contains the true distribution of $t$ and that the distribution of $t_{i}$ conditional on $x_{i}$ is in the proportional hazards family.

Our method mimics this approach but uses weighting instead of imputation. We begin with subdividing each individual's failure interval into non-overlapping subintervals. Using the set of all interval endpoints in the data set, those that fall into the individual's interval are then used as the cut points for the sub-intervals. This is a natural choice as the rank order of failure times is the only relevant information in a Cox model. Each sub-interval has an accompanying weight calculated from a parametric Weibull model (e.g., fit in SAS PROC LIFEREG) based on the current parameter estimates. The sum of the weights for each individual across their subintervals equals 1.0. For right-censored individuals, there are no sub-intervals and the accompanying weight is 1.0 .

Suppose there are $Q_{i}-1$ failure interval endpoints from other individuals contained in the $i^{t h}$ person's failure interval, resulting in $Q_{i}$ subintervals for that person. Denote the endpoints of the subintervals by $t_{i 0}, t_{i 1}, t_{i 2}, \ldots, t_{i Q_{i}}$, with $t_{i 0}=l_{i}$ and $t_{i Q_{i}}=u_{i}$. For 
convenience, we assign a pseudo failure time $t_{i q}^{*}$ using the lower bound value of the interval $t_{i q}^{*}=t_{i(q-1)}+0.001$ for each sub-interval $q$ for the $i^{t h}$ individual. The actual failure time within a sub-interval is not important as long as the ranks of failure times in the data set are preserved. This will create some tied observations in the weighted PH model and we use Breslow's method (1974) for handling them. Efron's method (1977) for handling ties is an alternative but takes more computation time. Denote the corresponding $q^{t h}$ weight for the $i^{t h}$ individual by $\omega_{i q}$. Then,

$$
\begin{gathered}
\omega_{i 1}=\left[S_{i}\left(l_{i}\right)-S_{i}\left(t_{i 1}\right)\right] /\left[S_{i}\left(l_{i}\right)-S_{i}\left(u_{i}\right)\right] \\
w_{i 2}=\left[S_{i}\left(t_{i 1}\right)-S_{i}\left(t_{i 2}\right)\right] /\left[S_{i}\left(l_{i}\right)-S_{i}\left(u_{i}\right)\right] \\
\ldots \\
\omega_{i 2}\left(Q_{i}\right)=\left[S_{i}\left(t_{i\left(Q_{i}-1\right)}\right)-S_{i}\left(u_{i}\right)\right] /\left[S_{i}\left(l_{i}\right)-S_{i}\left(u_{i}\right)\right] .
\end{gathered}
$$

It clearly follows that $\sum_{q=1}^{Q_{i}} \omega_{i q}=1$. The corresponding weighted log partiallikelihood is

$$
l(\beta, \theta)=\sum_{i=1}^{n} \sum_{q=1}^{Q_{i}} \omega_{i q} \delta_{i}\left\{X_{i}^{\prime}\left(t_{i q}^{*}\right) \beta-\log \left[\sum_{l \in R\left(t_{i q}^{*}\right)} \omega Y_{1}\left(t_{i q}^{*}\right) e^{x_{1}^{\prime}\left(t_{i q}^{*}\right) \beta}\right]\right\},
$$

where $R\left(t_{i q}^{*}\right)$ is the risk set at time $t_{i q}^{*}$ and $Y_{l}\left(t_{i q}^{*}\right)=I\left(T_{l} \geq t_{i q}^{*}\right)$.

An expanded data set will be generated with multiple lines for each individual corresponding to the sub-intervals. Each line will have the assigned pseudo failure time with the accompanying weight. Right-censored observations are handled in the usual manner. We iterate between estimating the baseline Weibull distribution and fitting the weighted PH model until the regression parameters converge. The linear predictor $\left(\boldsymbol{x}_{i}{ }^{\prime} \boldsymbol{\beta}_{\boldsymbol{A F T}}\right)$ is fixed as an offset when we update the estimates of the Weibull distribution and recalculate the weights.

\subsection{Implementation}

Specifically, our proposed approach is implemented as follows:

1. Center the covariates and fit a Weibull survival model (e.g., in SAS PROC LIFEREG) to obtain an initial estimate of $\beta_{A F T}$, denoted $\beta_{A F T, 0}$, and the survival distribution parameters (intercept $\Delta$ and scale $\sigma$ ). The Weibull distribution is chosen as it is in both the AFT and PH families.

2. Calculate the corresponding parameters for the PH model, denoted $\beta_{P H, 0}$, by dividing $-\hat{\beta}_{A F T, 0}$ by the estimate of the Weibull scale parameter $(\sigma)$.

3. Combine non-zero and non-missing $l_{i}$ and $u_{i}$ values and rank them in increasing order. 
4. Use the ranked interval endpoints to create sub-intervals for interval-censored individuals.

5. Calculate a weight for each left- or interval-censored observation based on the fitted Weibull model. Set the weight $=1.0$ for right-censored observations.

6. Create an expanded data set with multiple lines that includes the pseudo failure times and accompanying weights corresponding to the sub-intervals for each individual.

7. Fit a weighted PH model (e.g., in SAS PROC PHREG) to obtain an estimate of $\beta_{P H}$, denoted $\hat{\beta}_{P H}$.

8. Refit the Weibull survival model to obtain new estimates of the intercept and scale parameters but hold $X^{\prime} \hat{\beta}_{A F T}\left(=-X^{\prime} \hat{\beta}_{P H} \times \sigma\right)$ fixed with an OFFSET statement.

9. Return to step (5) and update the subinterval weights. Iterate until convergence, e.g. $\left|\hat{\beta}_{P H, j}-\hat{\beta}_{P H, j-1}\right|<\epsilon$ where $\epsilon=0.00001$.

\subsection{Parameter estimate standard errors}

Our proposed estimator $\hat{\beta}$ is asymptotically normally distributed:

$$
n^{\frac{1}{2}}\left(\hat{\beta}-\beta_{0}\right) \rightarrow N(0, V) .
$$

Standard software packages assume the weights in the weighted $\mathrm{PH}$ model are known. In comparison, the sub-interval weight for an individual here describes the probability that the exact failure time occurs within the sub-interval. The weight is a random variable subject to variability depending on the data and is estimated using the Weibull survival model. As a result, the usual standard errors of $\hat{\beta}$ from the weighted PH model will be underestimated. Let $S_{\beta}(\beta, \theta)$ and $U_{\theta}^{0}(\beta, \theta)$ denote the score equations obtained from the usual weighted PH model and the AFT interval-censored Weibull model, respectively. Similar to Satten et al. (1998) we propose a sandwich estimator for the variance-covariance matrix of parameter estimates $\left[\hat{\beta}^{\prime}, \hat{\theta}^{\prime}\right]^{\prime}$, which we will denote by $V$, with

$V=A^{\prime-1} \Psi A^{-1}$, where $-n A(\beta, \theta)$ is the expected value of the Jacobian matrix of the score equations given by

$$
\mathrm{A}(\beta, \theta)=\left[\begin{array}{ll}
A_{11}(\beta, \theta) & A_{12}(\beta, \theta) \\
A_{21}(\beta, \theta) & A_{22}(\beta, \theta)
\end{array}\right]
$$

that can be consistently estimated by

$$
\hat{A}^{n}(\hat{\beta}, \hat{\theta})=-\frac{1}{n}\left[\begin{array}{cc}
\frac{\partial}{\partial \beta} S_{\beta}(\hat{\beta}, \hat{\theta}) & \frac{\partial}{\partial \theta} S_{\beta}(\hat{\beta}, \hat{\theta}) \\
\frac{\partial}{\partial \beta} U_{\theta}^{o}(\hat{\beta}, \hat{\theta}) & \frac{\partial}{\partial \theta} U_{\theta}^{o}(\hat{\beta}, \hat{\theta})
\end{array}\right]
$$

Essentially, $A_{22}$ is the information matrix of $\hat{\theta}$ and thus can be easily estimated by 
inverting the variance-covariance matrix of $\hat{\theta}$ from the parametric Weibull model (e.g., SAS PROC LIFEREG). As for $A_{21}$, it is the derivative of the score equation $U_{\theta}^{0}(\beta, \theta)$ from the interval-censored Weibull model with respective to the PH model's $\beta$. Derivation of $A_{11}$ and $A_{12}$ is not straightforward as the score equations from the $\mathrm{PH}$ model $S_{\beta}(\beta, \theta)$ involve multiple observations (i.e., the sub-intervals) for each individual, and the corresponding weights also require estimation. See Appendix 1 for the score function for the parametric interval-censored Weibull survival model and Appendix 2 for the derivations of the consistent estimators for $A_{11}, A_{12}$ and $A_{21}$.

To develop an estimate for $\Psi$, we first write

$$
\widehat{\Psi}(\hat{\beta}, \hat{\theta})=\left[\begin{array}{ll}
\widehat{\Psi_{11}}(\hat{\beta}, \hat{\theta}) & \widehat{\Psi_{12}}(\hat{\beta}, \hat{\theta}) \\
\widehat{\Psi_{21}}(\hat{\beta}, \hat{\theta}) & \widehat{\Psi_{22}}(\hat{\beta}, \hat{\theta})
\end{array}\right]
$$

Following Satten et al. (1998),

$$
\begin{gathered}
\widehat{\psi}_{11}(\hat{\beta}, \hat{\theta})=\left(\frac{1}{n}\right) \sum_{i=1}^{n} \hat{\psi}_{i} \hat{\psi}_{i}^{\prime}, \\
\widehat{\psi}_{12}(\hat{\beta}, \hat{\theta})=\left(\frac{1}{n}\right) \sum_{i=1}^{n} \widehat{\psi}_{i} U_{i \theta}^{o}(\hat{\beta}, \hat{\theta})^{\prime},
\end{gathered}
$$

and

$$
\left.\widehat{\Psi}_{22}(\hat{\beta}, \hat{\theta})=\left(\frac{1}{n}\right) \sum_{i=1}^{n} U_{i \theta}^{o}(\hat{\beta}, \hat{\theta}) U_{i \theta}^{o} \hat{\beta}, \hat{\theta}\right)^{\prime},
$$

where $\hat{\psi}_{i}$ is the influence function for the $i^{\text {th }}$ subject in the PH model (Reid et al. (1985)),

$$
\widehat{\Psi}_{\mathrm{i}}=\left\{\begin{array}{l}
\sum_{q=1}^{Q_{i}} \omega_{i q} \hat{S}_{i q}^{P H} \text { if } \delta_{i}=1 \\
\hat{S}_{i}^{P H} \quad \text { if } \delta_{i}=0
\end{array}\right.
$$

In equation (3), $S_{i q}^{P H}$ is the individual contribution from the $q^{\text {th }}$ sub-interval of the $i^{\text {th }}$ subject to the partial derivative of the $\log$ likelihood with respect to $\beta$ under the $\mathrm{PH}$ model. Lin and Wei (1989) showed that the partial likelihood score function of $\beta$ for the PH model can be approximated by a sum of iid terms of the score residuals. Here we propose to estimate $S_{i q}^{P H}$ using score residuals. Specifically,

$$
\hat{S}_{i q}^{P H}=\delta_{i}\left\{x_{i}\left(t_{i q}^{*}\right)-\bar{x}\left(t_{i q}^{*}\right)\right\}-\sum_{j=1}^{n} \sum_{q=1}^{Q} \delta_{j} \frac{Y_{i}\left(t_{j q}^{*}\right) e^{x_{i}^{\prime}\left(t_{j q}^{*}\right) \widehat{\beta}}}{\sum_{l \in R\left(t_{j q}^{*}\right)} Y_{i}\left(t_{j q}^{*}\right) e^{x_{l}^{\prime}\left(t_{j q}^{*}\right) \widehat{\beta}}}\left\{x_{i}\left(t_{j q}^{*}\right)-\bar{x}\left(t_{j q}^{*}\right)\right\}
$$

where $\bar{x}\left(t_{. q}^{*}\right)=\frac{\sum_{l \in R\left(t_{q q}^{*}\right)} Y_{l}\left(t_{. q}^{*}\right) x_{l}\left(t_{. q}^{*}\right) e^{x^{\prime} l^{\prime}\left(t_{q}^{*}\right) \hat{\beta}}}{\sum_{l \in R\left(t_{q q}^{*}\right)} Y_{l}\left(t_{. q}^{*}\right) e^{x^{\prime}\left(t_{. q}^{*}\right) \hat{\beta}}}$, with.$=i$ or $j$. Hence $\hat{\psi}_{i}$ is the weighted 
version of the score residual vector $\hat{S}_{i q}^{P H}$. 


\section{Simulations}

We conducted two simulation trials to assess the performance of our proposed approach for fitting the Cox PH model to interval-censored failure time data. For the first set of simulations, we generated failure times with an underlying Weibull distribution as follows:

$$
P_{r}\left(T_{i} \geq t_{i}\right)=S\left(t_{i} ; \theta, \beta\right)=\left[S_{0}\left(t_{i} ; \theta\right)\right]^{\exp \left(x_{1 i} \beta_{1}+x_{2 i} \beta_{2}\right)},
$$

for $i=1, \ldots n$, where $S_{0}\left(t_{i} ; \theta\right)=\exp \left(-e^{\left(t_{i}-\Delta\right) / \sigma}\right), x_{1 i}=-0.5,0.5$ is binary with equal group sizes, and $x_{2 i} \sim(-1.0,1.0)$. The intercept parameter was specified as $\Delta=$ $\log (100)$, the scale parameter as $\sigma=0.5$, and $\beta_{1}=\beta_{2}=\log (2.0)=0.693$. Simulated data sets are of total size $n=100,500,1000$. Following Satten et al. (1998), interval censoring was conducted by starting an independent renewal process for each observation in each data set at 0 . The increments followed a lognormal distribution with mean 18.7 and standard error 209.4 for this simulation. A maximum number of 22 renewals was set. If one of the renewal intervals contained the true failure time, then this interval was used as the censoring interval; otherwise the observation was considered to be right censored at the last renewal time. Approximately $18 \%$ of the observations were right censored.

Two thousand data sets were generated for each sample size and were analyzed with our approach. For comparison, we analyzed the data with a parametric Weibull model using PROC LIFEREG in SAS (v 9.4). We also analyzed the exact survival times with the Cox PH model using PROC PHREG in SAS (v 9.4). Average parameter estimates over the 2000 data sets, average parameter estimate standard errors, and empirical parameter estimate standard errors were calculated for all approaches. 
Table 1: Simulation results using 2000 data sets with various analytic approaches. The failure times are generated with an underlying Weibull distribution as follows: $\operatorname{Pr}\left(T_{i} \geq t_{i}\right)=$ $S\left(t_{i} ; \theta, \beta\right)=\left[S_{0}\left(t_{i} ; \theta\right)\right]^{\exp \left(x_{1 i} \beta_{1}+x_{2 i} \beta_{2}\right)}$, where $S_{0}\left(t_{i} ; \theta\right)=\exp \left(-e^{\left(t_{i}-\Delta\right) / \sigma}\right)$ with $x_{1 i}=$ $-0.5,0.5$ (equal group size), and $x_{2 i} \sim U(-1.0,1.0)$. The interecept parameter was specified as $\Delta=\log (100)$, the scale parameter as $\sigma=0.5$, and $\beta_{1}=\beta_{2}=\log (2.0)=0.693$. Data sets are of total size $n=100,500,1000$.

\begin{tabular}{|c|c|c|c|c|c|c|c|}
\hline Method & Distribution & $\hat{\beta}_{1}^{a}$ & $\begin{array}{l}\text { s.e. } \\
\left(\hat{\beta}_{1}\right)^{b}\end{array}$ & $\operatorname{ese}\left(\hat{\beta}_{1}\right)^{c}$ & $\hat{\beta}_{2}^{a}$ & $\begin{array}{l}\text { s.e. } \\
\left(\hat{\beta}_{2}\right)^{b}\end{array}$ & $\operatorname{ese}\left(\hat{\beta}_{2}\right)^{c}$ \\
\hline \multicolumn{8}{|c|}{$\mathrm{N}=100$} \\
\hline Exact Cox $\mathrm{PH}^{\mathrm{d}}$ & & 0.707 & 0.216 & 0.219 & 0.712 & 0.192 & 0.200 \\
\hline Parametric ${ }^{\mathrm{e}}$ & Weibull & 0.722 & 0.255 & 0.269 & 0.725 & 0.220 & 0.234 \\
\hline Proposed $^{\mathrm{f}}$ & Weibull & 0.710 & 0.243 & 0.265 & 0.713 & 0.213 & 0.233 \\
\hline $\begin{array}{l}\text { Unadjusted standard } \\
\text { error }^{\mathrm{g}}\end{array}$ & Weibull & & 0.234 & & & 0.207 & \\
\hline \multicolumn{8}{|c|}{$\mathrm{N}=500$} \\
\hline Exact Cox PH & & 0.696 & 0.095 & 0.096 & 0.696 & 0.083 & 0.084 \\
\hline Parametric & Weibull & 0.699 & 0.112 & 0.115 & 0.698 & 0.098 & 0.101 \\
\hline Proposed & Weibull & 0.697 & 0.109 & 0.115 & 0.696 & 0.095 & 0.101 \\
\hline Unadjusted standard error & Weibull & & 0.103 & & & 0.090 & \\
\hline \multicolumn{8}{|c|}{$\mathrm{N}=1000$} \\
\hline Exact Cox PH & & 0.695 & 0.067 & 0.068 & 0.694 & 0.059 & 0.059 \\
\hline Parametric & Weibull & 0.694 & 0.079 & 0.079 & 0.694 & 0.069 & 0.070 \\
\hline Proposed & Weibull & 0.693 & 0.077 & 0.079 & 0.693 & 0.068 & 0.070 \\
\hline Unadjusted standard error & Weibull & & 0.072 & & & 0.064 & \\
\hline
\end{tabular}

${ }^{a}$ Average parameter estimate over 2000 simulated data sets.

${ }^{\mathrm{b}}$ Average standard error over 2000 simulated data sets.

${ }^{\mathrm{c}}$ Empirical standard error.

${ }^{\mathrm{d}} \mathrm{Cox}$ PH model on the exact failure times.

${ }^{\mathrm{e}}$ Parametric Weibull AFT model using PROC LIFEREG in SAS with parameters transformed to PH interpretation.

${ }^{\mathrm{f}}$ Proposed PH interval-censored failure time model.

${ }^{\mathrm{g}}$ Proposed PH interval-censored failure time model with standard errors unadjusted for estimation of weights.

The simulation results are presented in Table 1 . The average parameter estimates for $\beta_{1}$ and $\beta_{2}$ over the 2000 simulated data sets using our approach were, respectively, 0.710 and 0.713 for $n=100,0.697$ and 0.696 for $n=500$, and 0.692 and 0.693 for $n=1000$, comparable to the parameter estimates from both the parametric Weibull model and the Cox model on the exact times. As expected, the parameter estimates for all approaches were closer to 0.693 as $n$ increased. The proposed parameter estimate standard errors from Section 2.2 slightly underestimated the empirical parameter estimate standard errors for $\beta_{1}$ and $\beta_{2}$ for small sample sizes $(n=100)$, but were 
similar to them for the larger sample sizes $(n=500,1000)$. The naive parameter estimate standard errors without taking into account the adjustment due to estimating the weights underestimated the empirical parameter estimate standard errors by about $10 \%$.

We conducted a second simulation trial with misspecified data. We generated failure times with an underlying log-logistic distribution as follows:

$$
P_{r}\left(T_{i} \geq t_{i}\right)=S\left(t_{i} ; \theta, \beta\right)=\left[S_{0}\left(t_{i} ; \theta\right)\right]^{\exp \left(x_{1 i} \beta_{1}+x_{2 i} \beta_{2}\right)}
$$

for $i=1, \ldots, n$, with $S_{0}\left(t_{i} ; \theta\right)=\left(1+e^{\left(t_{i}-\Delta\right) / \sigma}\right)^{-1}$, and $x_{1}, x_{2}, \beta_{1}$, and $\beta_{2}$ as in the first set of simulations. The intercept parameter was specified as $\Delta=\log (100)$ and the scale parameter as $\sigma=0.15$. As such, the generated survival model still satisfies the $\mathrm{PH}$ assumption; however it does not belong to the PH family. Interval censoring was conducted in the same manner as for the first set of simulations except the lognormal distribution of the renewal process had mean 15 and standard error 109.8. A maximum number of 24 renewals was set and approximately $15 \%$ of the observations were right censored. See Figure 1 for a plot of the survival and hazard curves for the underlying survival distributions of the two sets of simulations. Two thousand data sets were generated again of size $n=100,500,1000$. The data sets were analyzed with the same analytic approaches as in the first set of simulations.
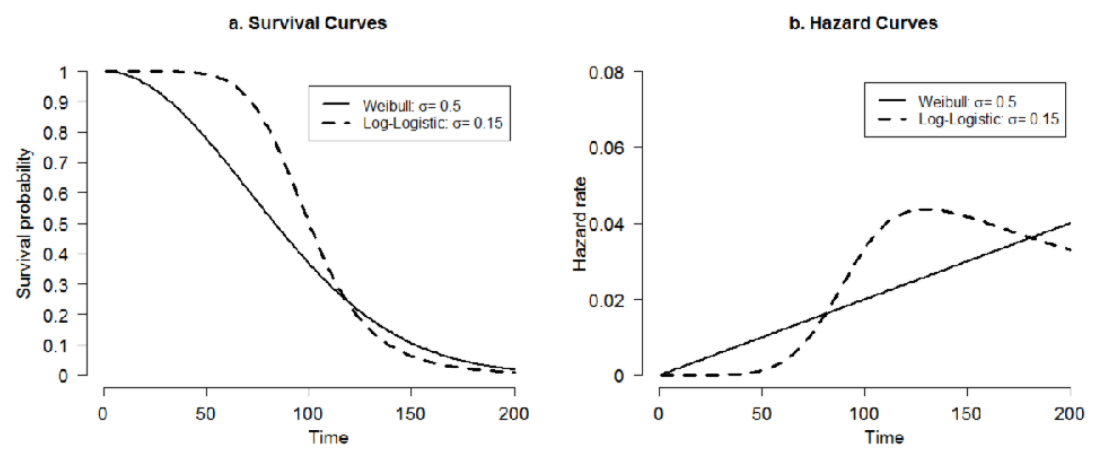

Figure 1: (a) Survival curves and (b) hazard curves, for the Weibull $(\operatorname{Pr}(T \geq t)=S(t ; \theta)=$ $\exp \left(-e^{(t-\Delta) / \sigma}\right)$ with $\Delta=\log (100)$, and $\left.\sigma=0.5\right)$ and Log-Logistic $(\operatorname{Pr}(T \geq t)=S(t ; \theta)=$ $\left(1+e^{(t-\Delta) / \sigma}\right)^{-1}$ with $\Delta=\log (100)$, and $\left.\sigma=0.15\right)$ distributions. The $\mathrm{x}$-axis is in original time scale before the log-transformation. 
Table 2: Simulation results using 2000 data sets with various analytic approaches. The logtransformed failure times are generated with an underlying log-logistic distribution as follows: $\left(\operatorname{Pr}\left(T_{i} \geq t_{i}\right)=S\left(t_{i} ; \theta, \beta\right)=\left[S_{0}\left(t_{i} ; \theta\right)\right]^{\exp \left(x_{1 i} \beta_{1}+x_{2 i} \beta_{2}\right)}\right.$, where $S_{0}\left(t_{i} ; \theta\right)=\left(1+e^{\left(t_{i}-\Delta\right) / \sigma}\right)^{-1}$ with $x_{1 i}=-0.5,0.5$ (equal group size), and $x_{2 i} \sim U(-1.0,1.0)$. The interecept parameter was specified as $\Delta=\log (100)$, the scale parameter as $\sigma=0.15$, and $\beta_{1}=\beta_{2}=\log (2.0)=0.693$.

Data sets are of total size $n=100,500,1000$.

\begin{tabular}{|c|c|c|c|c|c|c|c|}
\hline Method & Distribution & $\hat{\beta}_{1}^{a}$ & $\begin{array}{l}\text { s.e. } \\
\left(\hat{\beta}_{1}\right)^{b}\end{array}$ & $\operatorname{ese}\left(\hat{\beta}_{1}\right)^{c}$ & $\hat{\beta}_{2}^{a}$ & $\begin{array}{l}\text { s.e. } \\
\left(\hat{\beta}_{2}\right)^{b}\end{array}$ & $\operatorname{ese}\left(\hat{\beta}_{2}\right)^{c}$ \\
\hline \multicolumn{8}{|c|}{$\mathrm{N}=100$} \\
\hline Exact Cox $\mathrm{PH}^{\mathrm{d}}$ & & 0.707 & 0.216 & 0.218 & 0.712 & 0.192 & 0.198 \\
\hline Parametric $^{\mathrm{e}}$ & Weibull & 0.836 & 0.281 & 0.319 & 0.842 & 0.249 & 0.287 \\
\hline Proposed $^{f}$ & Weibull & 0.712 & 0.269 & 0.276 & 0.723 & 0.242 & 0.251 \\
\hline $\begin{array}{l}\text { Unadjusted standard } \\
\text { error }^{\mathrm{g}}\end{array}$ & Weibull & & 0.232 & & & 0.206 & \\
\hline \multicolumn{8}{|c|}{$\mathrm{N}=500$} \\
\hline Exact Cox PH & & 0.697 & 0.094 & 0.096 & 0.697 & 0.083 & 0.084 \\
\hline Parametric & Weibull & 0.812 & 0.122 & 0.135 & 0.813 & 0.106 & 0.126 \\
\hline Proposed & Weibull & 0.698 & 0.126 & 0.116 & 0.699 & 0.114 & 0.106 \\
\hline Unadjusted standard error & Weibull & & 0.102 & & & 0.089 & \\
\hline \multicolumn{8}{|c|}{$\mathrm{N}=1000$} \\
\hline Exact Cox PH & & 0.694 & 0.067 & 0.069 & 0.694 & 0.059 & 0.057 \\
\hline Parametric & Weibull & 0.809 & 0.086 & 0.097 & 0.808 & 0.075 & 0.084 \\
\hline Proposed & Weibull & 0.696 & 0.092 & 0.084 & 0.694 & 0.084 & 0.072 \\
\hline Unadjusted standard error & Weibull & & 0.072 & & & 0.063 & \\
\hline
\end{tabular}

${ }^{a}$ Average parameter estimate over 2000 simulated data sets.

${ }^{\mathrm{b}}$ Average standard error over 2000 simulated data sets.

${ }^{\mathrm{c}}$ Empirical standard error.

${ }^{\mathrm{d}} \mathrm{Cox} \mathrm{PH}$ model on the exact failure times.

${ }^{\mathrm{e}}$ Parametric Weibull AFT model using PROC LIFEREG in SAS with parameters transformed to PH interpretation.

${ }^{\mathrm{f}}$ Proposed PH interval-censored failure time model.

${ }^{\mathrm{g}}$ Proposed PH interval-censored failure time model with standard errors unadjusted for estimation of weights.

The simulation results are presented in Table 2 . The average parameter estimates for $\beta_{1}$ and $\beta_{2}$ over the 2000 simulated data sets using our approach were, respectively, 0.712 and 0.723 for $n=100,0.698$ and 0.699 for $n=500$, and 0.696 and 0.694 for $n=1000$, again comparable to the parameter estimates from the Cox model on the exact times for $n=500$ and $n=1000$. For $n=100$, the parameter estimates from the proposed approach only slightly overestimate those from the Cox model on the exact times. The proposed parameter estimate standard errors were again very similar to the 
empirical parameter estimate standard errors for $\beta_{1}$ and $\beta_{2}$. Parameter estimates from the misspecified parametric Weibull model were severely biased as expected, and indicate the departure of the underlying log-logistic distribution from a Weibull distribution. See Figure 2 for box plots of the parameter estimates of $\beta_{1}$ and $\beta_{2}$ from this set of simulations. All simulations were conducted via SAS IML (2012).

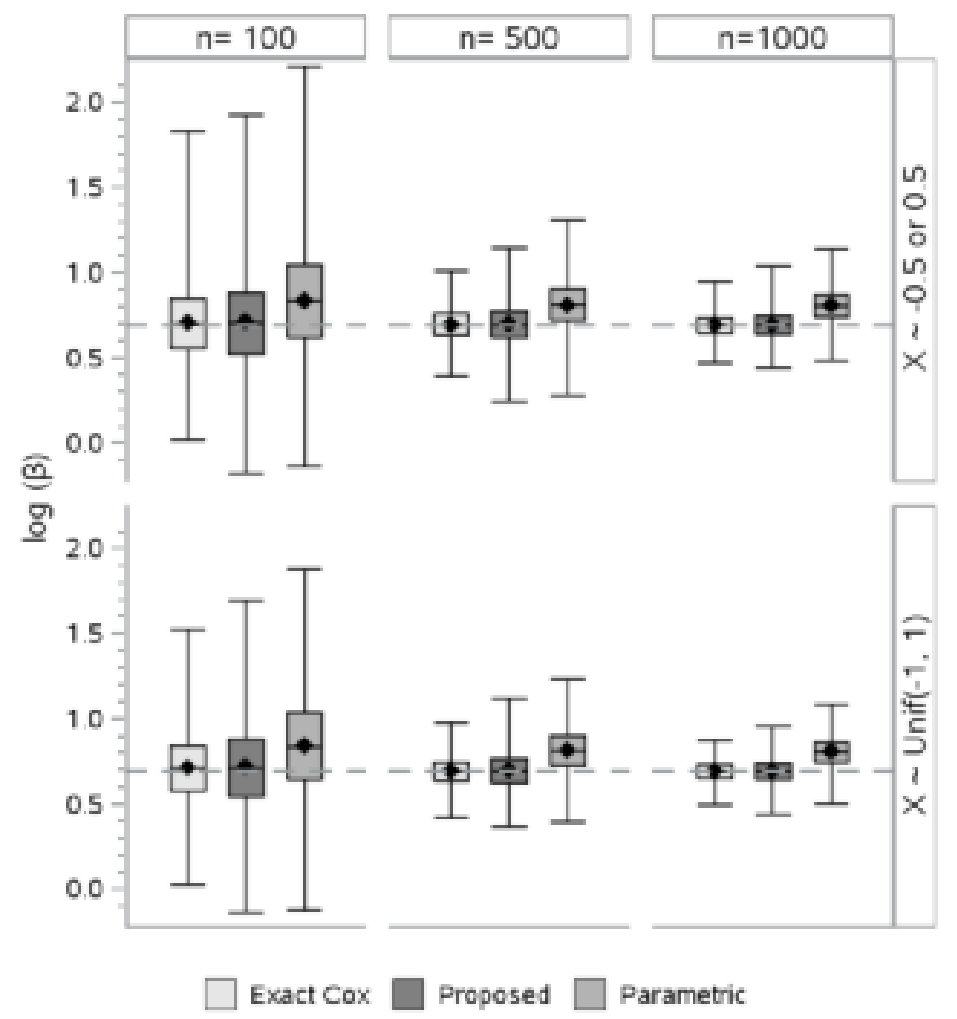

Figure 2: Boxplots of the simulation results for Table 2 (misspecified model) for all three sample sizes and both marginal parameters $\left(\beta_{1}\right.$ and $\left.\beta_{2}\right)$. The solid diamond represents the mean value, and the horizontal lines from the bottom to the top represent the minimum, 25 th percentile, median, 75 th percentile, and maximum values.

Exact Cox: PH model on the exact failure times. Proposed : Our proposed PH interval-censored failure time model. Parametric: Weibull AFT model with parameter transformed to PH interpretation.

\subsection{Comparison with Satten et al.'s approach}

We conducted another simulation trial to assess the performance of our proposed approach with Satten et al.'s PH interval-censored failure time model (Satten et al. (1998)). For comparison we generated the data for correctly-specified and mis-specified models following Satten et al. (1998). For the former model, we generated failure times 
with an underlying Weibull distribution and one covariate as follows:

$$
P_{r}\left(T_{i} \geq t_{i}\right)=S\left(t_{i} ; \theta, \beta\right)=\left[\exp \left(-e^{\left(t_{i}-\Delta\right) / \sigma}\right)\right]^{\exp \left(x_{i} ; \beta\right)}
$$

for $i=1, \ldots n$. The intercept parameter was specified as $\Delta=\log (100)$, the scale parameter as $\sigma=0.5$, and $x_{i}=-0.5,0.5$ is binary with equal group sizes and then $x_{i} \sim U(-1.0,1.0)$. The regression parameter $\beta=\log (2.0)=0.693$. For the misspecified model, we generated failure times with an underlying log-logistic distribution as follows:

$$
\left.P_{r}\left(T_{i} \geq t_{i}\right)=S\left(t_{i} ; \theta, \beta\right)=\left[1+e^{\left(t_{i}-\Delta\right) / \sigma}\right)\right]^{\exp \left(x_{i} ; \beta\right)}
$$

for $i=1, \ldots n$. The intercept parameter was specified as $\Delta=\log (100)$ and the scale parameter as $\sigma=0.25$. The covariate $x_{i}$ and the regression parameter $\beta$ are the same as for the Weibull model. Interval censoring was conducted for each set of simulations in the same manner as our first set of simulations. Approximately $24 \%$ (17\%) of the observations were right censored for the simulation with the continuous (binary) covariate.

Following Satten et al. (1998), simulated data sets are of total size $n=500$ for both sets of simulations. Only 500 data sets were generated for each covariate choice and simulation because of the lengthy computation time required to fit Satten et al.'s model (1998). We used 400 stochastic approximation steps with a block size of 50, and 75 'burn-in' steps with Satten et al.'s approach (1998). See Satten et al. (1998) for details. Data sets were analyzed with our approach and Satten et al.'s method (1998). For comparison, we also analyzed the exact survival times with the Cox PH model using PROC PHREG in SAS (v 9.4). Average parameter estimates over the 500 data sets, average parameter estimate standard errors, and empirical parameter estimate standard errors were calculated for all approaches. The simulation results are presented in Tables 3 (correctly specified) and 4 (incorrectly specified). 
Table 3: Simulation results using 500 data sets with Satten et al.'s (1998) PH interval-censored failure time model and the proposed approach. The failure times are generated with an underlying Weibull distribution as follows: $\operatorname{Pr}\left(T_{i} \geq t_{i}\right)=S\left(t_{i} ; \boldsymbol{\theta}, \boldsymbol{\beta}\right)=\left[S_{0}\left(t_{i} ; \boldsymbol{\theta}\right)\right]^{\exp \left(x_{i} \beta\right)}$, where

$S_{0}\left(t_{i} ; \boldsymbol{\theta}\right)=\exp \left(-e^{\left(t_{i}-\Delta\right) / \sigma}\right)$ with $x_{i}=-0.5,0.5$ (equal group size) or $x_{i} \sim U(-1.0,1.0)$. The intercept parameter was specified as $\Delta=\log (100)$, the scale parameter as $\sigma=0.5$, and $\beta$ varying. Data sets are of total size $n=500$.

\begin{tabular}{cclccc}
\hline Covariate & \multicolumn{1}{c}{$\beta$} & Method & $\hat{\beta}_{1}^{a}$ & s.e. $\left(\hat{\beta}_{1}\right)^{b}$ & ese $\left(\hat{\beta}_{1}\right)^{c}$ \\
\hline$x=-0.5,0.5$ & $\ln (1.5)=0.405$ & Exact Cox PH $^{\mathrm{d}}$ & 0.415 & 0.092 & 0.089 \\
& & Satten $^{\mathrm{e}}$ & 0.417 & 0.111 & 0.103 \\
& Proposed $^{\mathrm{f}}$ & 0.416 & 0.108 & 0.103 \\
& $\ln (2.0)=0.693$ & Exact Cox PH & 0.699 & 0.095 & 0.088 \\
& Satten & 0.700 & 0.114 & 0.107 \\
& & Proposed & 0.698 & 0.109 & 0.107 \\
& $\ln (2.5)=0.916$ & Exact Cox PH & 0.918 & 0.098 & 0.094 \\
& & Satten & 0.923 & 0.119 & 0.114 \\
& & Proposed & 0.921 & 0.110 & 0.114 \\
\hline$x \sim U(-1.0,1.0)$ & $\ln (1.5)=0.405$ & Exact Cox PH & 0.409 & 0.092 & 0.090 \\
& & Satten & 0.408 & 0.098 & 0.097 \\
& & Proposed & 0.407 & 0.096 & 0.096 \\
& $\ln (2.0)=0.693$ & Exact Cox PH & 0.694 & 0.094 & 0.096 \\
& & Satten & 0.701 & 0.104 & 0.096 \\
& & Proposed & 0.700 & 0.098 & 0.096 \\
& $\ln (2.5)=0.916$ & Exact Cox PH & 0.920 & 0.098 & 0.096 \\
& & Satten & 0.914 & 0.110 & 0.105 \\
& & Proposed & 0.913 & 0.100 & 0.104 \\
\hline
\end{tabular}

${ }^{\text {a }}$ Average parameter estimate over 500 simulated data sets.

${ }^{\mathrm{b}}$ Average parameter estimate standard error over 500 simulated data sets.

${ }^{\mathrm{c}}$ Empirical standard error.

${ }^{\mathrm{d}}$ Cox PH model on the exact failure times.

e Satten et al.'s (1998) PH interval-censored failure time model.

${ }^{\mathrm{f}}$ Proposed PH interval-censored failure time model. 
Table 4: Simulation results using 500 data sets with Satten et al.'s (1998) PH interval-censored failure time model and the proposed approach. The log-transformed failure times are generated with an underlying log-logistic distribution as follows: $\operatorname{Pr}\left(T_{i} \geq t_{i}\right)=S\left(t_{i} ; \boldsymbol{\theta}, \boldsymbol{\beta}\right)=\left[S_{0}\left(t_{i} ; \boldsymbol{\theta}\right)\right]^{\exp \left(x_{i} \beta\right)}$, where $S_{0}\left(t_{i} ; \boldsymbol{\theta}\right)=\left(1+e^{\left(t_{i}-\Delta\right) / \sigma}\right)^{-1}$ with $x_{i}=-0.5,0.5$ (equal group size) or $x_{i} \sim U(-1.0,1.0)$. The intercept parameter was specified as $\Delta=\log (100)$, the scale parameter as $\sigma=0.25$, and $\beta$ varying. Data sets are of total size $n=500$.

\begin{tabular}{cclccc}
\hline Covariate & \multicolumn{1}{c}{$\beta$} & Method & $\hat{\beta}_{1}^{a}$ & s.e. $\left(\hat{\beta}_{1}\right)^{b}$ & ese $\left(\hat{\beta}_{1}\right)^{c}$ \\
\hline$x=-0.5,0.5$ & $\ln (1.5)=0.405$ & Exact Cox PH $^{\mathrm{d}}$ & 0.412 & 0.092 & 0.093 \\
& & Satten $^{\mathrm{e}}$ & 0.418 & 0.111 & 0.109 \\
& Proposed $^{\mathrm{f}}$ & 0.418 & 0.109 & 0.110 \\
& $\ln (2.0)=0.693$ & Exact Cox PH & 0.691 & 0.095 & 0.097 \\
& & Satten & 0.694 & 0.118 & 0.116 \\
& Proposed & 0.696 & 0.113 & 0.116 \\
& $\ln (2.5)=0.916$ & Exact Cox PH & 0.929 & 0.098 & 0.102 \\
& & Satten & 0.936 & 0.128 & 0.115 \\
& & Proposed & 0.938 & 0.117 & 0.115 \\
\hline$x \sim U(-1.0,1.0)$ & $\ln (1.5)=0.405$ & Exact Cox PH & 0.412 & 0.092 & 0.093 \\
& & Satten & 0.409 & 0.099 & 0.097 \\
& & Proposed & 0.410 & 0.097 & 0.097 \\
& $\ln (2.0)=0.693$ & Exact Cox PH & 0.697 & 0.095 & 0.096 \\
& & Satten & 0.703 & 0.109 & 0.099 \\
& & Proposed & 0.705 & 0.102 & 0.099 \\
& $\ln (2.5)=0.916$ & Exact Cox PH & 0.918 & 0.098 & 0.098 \\
& & Satten & 0.919 & 0.118 & 0.108 \\
& & Proposed & 0.921 & 0.107 & 0.108 \\
\hline
\end{tabular}

${ }^{a}$ Average parameter estimate over 500 simulated data sets.

${ }^{\mathrm{b}}$ Average parameter estimate standard error over 500 simulated data sets.

${ }^{\mathrm{c}}$ Empirical standard error.

${ }^{\mathrm{d}} \mathrm{Cox} \mathrm{PH}$ model on the exact failure times.

${ }^{\mathrm{e}}$ Satten et al.'s (1998) PH interval-censored failure time model.

${ }^{\mathrm{f}}$ Proposed PH interval-censored failure time model.

The parameter estimates using the proposed approach and Satten et al.'s method (1998) are nearly identical for both the Weibull and log-logistic models regardless of covariate type. The largest difference between the parameter estimates of the two methods across the 3000 simulated data sets is 0.0064 (0.0061) for the Weibull (loglogistic) model simulation. As a consequence of this the empirical standard error estimates are essentially the same for the two methods. The parameter estimate standard errors for the proposed approach are closer than Satten et al.'s standard errors to the empirical standard errors for the majority of simulations. On average a simulation took approximately 44 hours using Satten et al.'s method (1998) and less than three hours using the proposed method. 


\section{Illustrative examples}

\subsection{Breast cancer data}

Here we illustrate our approach with two examples. A retrospective study of 94 women was conducted on the risk of breast cosmetic deterioration after tumorectomy. The interval-censored failure data are presented in Table 5 of Finkelstein and Wolfe (1985). The women received either radiation therapy $(x=-0.5, n=46)$ or radiation plus chemotherapy ( $x=0.5, n=48$ ) and visited the clinic every four to six months. No woman was seen after 48 months and 38 women never experienced the outcome (right censored). Finkelstein analyzed the data with a semiparametric PH model (Finkelstein (1986)). We also present analysis using an AFT Weibull model, our proposed approach, Sun's nonparametric test for interval-censored survival data (Sun (1996)), and Sun, Zhao, and Zhao's generalized log-rank test (2005). The results are presented in Table 5 . Time to breast cosmetic deterioration was significantly shorter ( $p$ value $<0.01)$ in the radiation plus chemotherapy group than the radiation therapy alone group according to all analytic approaches.

Table 5: Analyses of cosmetic deterioration (retraction) data for 94 early breast cancer patients treated with radiotherapy and chemotherapy versus radiotherapy alone.

\begin{tabular}{llccc}
\hline Method & Type & $\hat{\beta}$ & s.e. $(\hat{\beta})$ & p-value \\
\hline Parametric $^{\mathrm{a}}$ & & 0.917 & 0.283 & 0.0012 \\
Proposed $^{\mathrm{b}}$ & & 0.903 & 0.280 & 0.0013 \\
Nonparametric & Sun $^{\mathrm{c}}$ & & & 0.0068 \\
& Sun, Zhao, and Zhao $^{\mathrm{d}}$ & & & 0.0070 \\
Semiparametric & Finkelstein $^{\mathrm{e}}$ & & & 0.0064 \\
\hline
\end{tabular}

${ }^{\text {a }}$ Parametric Weibull AFT model using PROC LIFEREG in SAS with parameters transformed to PH interpretation.

${ }^{\mathrm{b}}$ Proposed PH interval-censored failure time model.

${ }^{\mathrm{c}}$ Sun's nonparametric test (1996) using PROC ICLIFETEST in SAS.

${ }^{\mathrm{d}}$ Sun, Zhao, and Zhao's generalized log-rank test (2005) using PROC ICLIFETEST in SAS.

${ }^{\mathrm{e}}$ Finkelstein's PH model for interval-censored failure time data (1986) using PROC ICLIFETEST in SAS. 


\subsection{ICU hyperlactemia data}

Elevated lactate levels are frequently observed in patients undergoing mitral valve surgery upon admission to an intensive care unit (ICU). Hyperlactemia is defined as a blood lactate level $\geq 2 \mathrm{mmol} / \mathrm{l}$ and is linked to poor postoperative prognosis. The objective of this analysis is to identify factors associated with lactate clearance $(<2.0$ $\mathrm{mmol} / \mathrm{l}$.).

We analyzed data from 907 heart surgery patients with a mean age of $62(\mathrm{SD}=14)$ years and a median cardiopulmonary bypass (CPB) time of 144 [IQR: $(117,180)$ ] minutes. Sixty-six percent of the patients had an American Society of Anesthesiologists (ASA) physical status score $\geq 4$ (indicating patients with severe systemic disease that was a constant threat to life or were not expected to survive without the operation) and $3.5 \%$ had history of dialysis. All patients had lactate measured routinely at the time of ICU admission. The timing of repeated lactate measures was at the discretion of the managing critical care team. A 2 hour time interval was considered standard practice, and more frequent lactate measurements were obtained if clinically indicated.

Preliminary work found that an initial lactate threshold of $\geq 7 \mathrm{mmol} / \mathrm{l}$ was associated with significant increased 30-day mortality: $15 \%$ vs $1.3 \%$.

We analyzed the data with our proposed model and a parametric AFT Weibull model. The outcome was time to lactate clearance. Patients with initial lactate level below $2 \mathrm{mmol} / \mathrm{l}$ were left censored (28.6\%). Their observations were treated as the usual interval-censored ones, but with the weight for the first subinterval being $\left[1.0-S_{i}\left(t_{i 1}\right)\right] /\left[1.0-S_{i}\left(u_{i}\right)\right]$, i.e., $S_{i}\left(l_{i}\right)=1.0$. Patients without lactate clearance at 24 hours after ICU arrival were right censored $(6.5 \%)$. The results are presented in Table 6. Our proposed model and the parametric Weibull model suggest that longer CPB time and higher initial lactate levels are associated with longer time for lactate clearance. History of dialysis is associated with faster clearance, which could be a result of more aggressive peri-operative management. However, our model also suggests significant associations between older age and ASA physical status $\geq 4$ with longer clearance time. In general, the parameter estimates from our proposed model were slightly larger in magnitude, resulting in more significant $p$-values. 
Table 6: Analyses of ICU hyperlactemia data for 907 patients undergoing mitral valve surgery.

The outcome is time to lactate clearance $(\leq 2.0 \mathrm{mmol} / \mathrm{l})$.

\begin{tabular}{|c|c|c|c|c|c|c|}
\hline \multirow[b]{2}{*}{ Variable } & \multicolumn{3}{|c|}{ Proposed Model $^{\mathrm{a}}$} & \multicolumn{3}{|c|}{ Parametric Model ${ }^{\mathrm{b}}$} \\
\hline & $\hat{\beta}_{P H}$ & s.e. $\left(\hat{\beta}_{P H}\right)$ & p-value & $\hat{\beta}_{P H}$ & s.e. $\left(\hat{\beta}_{P H}\right)$ & p-value \\
\hline Age (years) & -0.008 & 0.002 & 0.001 & -0.003 & 0.003 & 0.253 \\
\hline History of dialysis (yes vs. no) & 0.485 & 0.195 & 0.013 & 0.505 & 0.195 & 0.010 \\
\hline Cardiopulmonary bypass time (minutes) & -0.005 & 0.001 & $<0.001$ & -0.004 & 0.001 & $<0.001$ \\
\hline ASA physical status $\geq 4$ (yes vs. no) $^{c}$ & -0.187 & 0.076 & 0.014 & -0.133 & 0.076 & 0.081 \\
\hline $\begin{array}{l}\text { Initial lactate in } \mathrm{ICU} \geq 7 \mathrm{mmol} / \mathrm{l} \text { (yes } \\
\text { vs. no) }\end{array}$ & -1.230 & 0.158 & $<0.001$ & -0.937 & 0.174 & $<0.001$ \\
\hline
\end{tabular}

${ }^{\text {a }}$ Proposed PH interval-censored failure time model.

${ }^{\mathrm{b}}$ Parametric Weibull AFT model using PROC LIFEREG in SAS with parameters transformed to $\mathrm{PH}$ interpretation.

${ }^{c}$ ASA: American Society of Anesthesiology physical status classification. ASA $\geq 4$ indicates patients with life threatening disease.

\section{Discussion}

Interval-censored failure data often arise in longitudinal studies in which subjects are assessed only periodically for the response of interest (Sun (2006)). The time when the event of interest occurs is not directly observed but is known to take place within some time interval. We propose a $\mathrm{PH}$ model for analysis of such data that have overlapping time intervals. We subdivide each individual's failure interval into nonoverlapping sub-intervals. Within each sub-interval, a pseudo failure observation is assigned, and later weighted in the PH model. The weight of the sub-interval is derived using a parametric Weibull model to reflect the probability that the individual's failure time occurred in that sub-interval. We use a robust estimator for the variancecovariance matrix of parameter estimates to further protect against misspecification of the failure time model. Simulation results demonstrate apparently unbiased parameter estimation for the correctly specified Weibull model and minimal bias for a misspecified log-logistic model. The proposed approach reduces to the usual Cox PH model if there is no overlap in failure intervals between observations. These programs are written in SAS IML (2012) and are available from the authors upon request and at https://www.researchgate.net/profile/John_Williamson 20.

In the situation when follow-up visits are all scheduled at the same time for each patient, resulting in non-overlapping sub-intervals for an individual, subjects who fail in the same interval will be tied. Consequently, if there are only a few available intervals, the data set might contain a considerable amount of ties. Our approach would not be advantageous and these data would be better analyzed using a proportional hazards method for grouped survival data (e.g., Fahrmeir and Tutz (1994), Scheike and Kold 
Jensen (1997)). Also both our proposed method and Satten el al.'s method (1998) assume that censoring intervals are independent of the covariates $x$ as with the usual PH model. It would require re-development of both score functions $\left(S_{\beta}(\beta, \theta)\right.$ and $\left.U_{\theta}^{0}(\beta, \theta)\right)$ and the matrices $A$ and $\Psi$ to allow for this dependency using the proposed approach.

The parameter estimates using the proposed approach are essentially the same as those using Satten et al.'s method (1998) in the simulation results in Tables 3 and 4. However, the corresponding parameter estimate standard errors using the proposed approach were in general closer to the empirical standard errors. Our approach is also computationally simpler as it does not require imputation nor bootstrapping, which is especially advantageous for large data sets or model building. Following Satten et al. (1998), each of the simulated data sets required fitting 20,000 Cox PH models. Additionally, Satten et al.'s method (1998) involves random number generation for imputation implying that each subsequent analysis of the same data set will produce slightly different results. In contrast the proposed approach produces the same results.

Assessment of the proportional hazards assumption should be conducted for interval-censored proportional hazards regression as for the usual right-censored proportional hazards regression model. For a categorical covariate, the simplest and most direct method to accomplish this is by plotting the survival functions of subjects with the same covariate value on the same graph (Sun (2006)). A nonparametric estimate of the survival function for interval-censored survival data can be fit using the efficient EMICM algorithm (Wellner and Zhan (1997)) in available software such as PROC ICLIFETEST in SAS (Guo et al. (2014)). For a continuous covariate, one can group the values into a small number of distinct intervals and apply the above approach (Sun (2006)).

\section{Acknowledgements}

This paper was prepared by John Williamson in his personal capacity. The opinions expressed in this article are the author's own and do not reflect the view of the Centers for Disease Control and Prevention, the Department of Health and Human Services, or the United States government. 


\section{References}

[1] Alkarni, S.H. (2016). Generalized extended Weibull power series family of distributions. Journal of Data Science 14, 415-440.

[2] Breslow, N. (1974). Covariance analysis of censored survival data. Biometrics 30, 89-99.

[3] Cox, D.R. (1972). Regression models and life tables. Journal of the Royal Statistical Society, Series B 20, 187-220.

[4] Cox, D.R. (1975). Partial likelihood. Biometrika 62, 269-276.

[5] Diamond, I.D., McDonald, J.W. and Shah, I.H. (1986). Proportional hazards models for current-status data: application to the study of differentials in age at weaning in Pakistan. Demography 23, 607-620.

[6] Efron, B. (1977). The efficiency of Cox's likelihood function for censored data. Journal of the American Statistical Association 72, 557-565.

[7] Fahrmeir, L. and Tutz, G. (1994). Multivariate Statistical Modelling Based on Generalised Linear Models. Springer, New York.

[8] Finkelstein, D.M. (1986). A proportional hazards model for interval-censored failure time data. Biometrics 42, 845-854.

[9] Finkelstein, D.M. and Wolfe, R.A. (1985). A semiparametric model for regression analysis of interval-censored failure time data. Biometrics 41, 933-945.

[10] Grummer-Strawn, L.M. (1993). Regression analysis of current-status data: An application to breast-feeding. Journal of the American Statistical Association 88, 758765.

[11] Guo, C., So, Y. and Johnston, G. (2014). Analyzing interval-censored survival data with the ICLIFETEST procedure. SAS Global Forum. SAS Institute, Inc.: Cary, NC, Paper 279-2014.

[12] Heller, G. (2011). Proportional hazards regression with interval censored data using an inverse probability weight. Lifetime Data Analysis 17, 373-385.

[13] Huang, J. (1996). Efficient estimation for the proportional type I interval censored data. The Annals of Statistics 24, 540-568.

[14] Lin, D.Y. and Wei, L.J. (1989). The robust inference for the Cox proportional hazards model. Journal of American Statistical Association 84, 1074-1078. 
[15] Lin, X., Cai, B., Wang, L. and Zhang, Z. (2015). A Bayesian proportional hazards model for general interval-censored data. Lifetime Data Analysis 21, 470-490.

[16] Murphy, S.A. and van der Vaart, A.W. (1997). Semiparametric likelihood ratio inference. Annals of Statistics 25, 1471-1509.

[17] Mustafa, A., El-Desouky, B.S. and AL-Garash, S. (2016). The Weibull generalized flexible Weibull extension distribution. Journal of Data Science 14, 453-478.

[18] Peto, R. and Peto, J. (1972). Asymptotically efficient rank invariant test procedures. Journal of the Royal Statistical Society, Series A 5, 185-207.

[19] $\mathrm{Pu}$, S., Oluyede, B.O., Qiu, Y., and Linder, D. (2016). A generalized class of exponentiated modified Weibull distribution with applications. Journal of Data Science 14, 585-614.

[20] Reid, N. and Crepeau, H. (1985). Influence functions for proportional hazards regression. Biometrika 72, 1-9.

[21] SAS Institute, Inc. (2012). SAS/IML Software: Changes and Enhancements through Release 12.1. SAS Institute, Inc.: Cary, North Carolina.

[22] Satten, G.A. (1996). Rank-based inference in the proportional hazards model for interval censored data. Biometrika 83, 355-370.

[23] Satten, G.A., Datta, S. and Williamson, J.M. (1998). Inference based on imputed failure times for the proportional hazards model with interval-censored data. Journal of the American Statistical Association 93, 318-327.

[24] Scheike, T.H. and Kold Jensen, T. (1997). A discrete survival model with random effects: an application to time to pregnancy. Biometrics 53, 318-329.

[25] Sun, J. (1996). A nonparametric test for interval-censored failure time data with application to AIDS studies. Statistics in Medicine 15, 1387-1395.

[26] Sun, J. (2006). The Statistical Analysis of Interval-Censored Failure Time Data. Springer Science+Business Media, Inc., New York.

[27] Sun, J., Zhao, Q. and Zhao, X. (2005). Generalized log-rank test for interval-censored failure time time data. Scandinavian Journal of Statistics 32, 49-57.

[28] Wellner, J.A. and Zhan, Y. (1997). A hybrid algorithm for computation of the nonparametric maximum likelihood estimator from censored data. Journal of the American Statistical Association 92, 945-959. 
[29] Zhao, Q. and Sun, J. (2004). Generalized log-rank test for mixed interval-censored failure time data. Statistics in Medicine 23, 1621-1629. 


\section{Appendix 1}

\section{Interval-Censored Weibull Model Score Equations with Respect to $\beta_{P H}$}

Assume a Weibull model in SAS PROC LIFEREG notation (Accelerated Failure Time Model) for interval-censored data. Let the lower $\left(l_{i}\right)$ and upper $\left(u_{i}\right)$ ends of the survival interval be log-transformed. Let $z_{i}=\left(\frac{1}{\sigma}\right)\left(u_{i}-\Delta-x_{i}^{\prime} \beta_{A F T}\right)=\frac{u_{i}}{\sigma}-\frac{\Delta}{\sigma}+x_{i}^{\prime} \beta_{P H}$ and $v_{i}=\left(\frac{1}{\sigma}\right)\left(l_{i}-\Delta-x_{i}^{\prime} \beta_{A F T}\right)=\frac{l_{i}}{\sigma}-\frac{\Delta}{\sigma}+x_{i}^{\prime} \beta_{P H}$, with $S\left(z_{i}\right)=\exp \left\{-\exp \left(z_{i}\right)\right\}$, $f\left(z_{i}\right)=\left(\frac{1}{\sigma}\right) \exp \left(z_{i}\right) \exp \left\{-\exp \left(z_{i}\right)\right\}$ and the corresponding survivor and pdf functions for $v_{i}$. For clarity, we define censoring status with 3 random variables as follows: $\lambda_{1 i}=1$ if the $i^{\text {th }}$ observation is left censored and 0 otherwise; $\lambda_{2 i}=1$ if the $i^{\text {th }}$ observation is interval censored and 0 otherwise, and $\lambda_{3 i}=1$ if both $\lambda_{1 i}=0$ and $\lambda_{2 i}=0$ for right censoring.

The log-likelihood function for such data is:

$$
\begin{aligned}
\log \mathrm{L}= & \sum_{i=1}^{N} \lambda_{1 i} \log \left(F\left(z_{i}\right)\right)+\sum_{i=1}^{N} \lambda_{2 i} \log \left[F\left(z_{i}\right)-F\left(v_{i}\right)\right]+\sum_{i=1}^{N} \lambda_{3 i} \log \left(S\left(v_{i}\right)\right) \\
= & \sum_{i=1}^{N} \lambda_{1 i} \log \left(1-\exp \left(-\exp \left(z_{i}\right)\right)\right) \\
& \quad+\sum_{i=1}^{N} \lambda_{2 i} \log \left[\exp \left(-\exp \left(v_{i}\right)-\exp \left(-\exp \left(z_{i}\right)\right)\right]\right. \\
& \quad+\sum_{i=1}^{N} \lambda_{3 i} \log \left(\exp \left(-\exp \left(v_{i}\right)\right)\right) \\
= & \sum_{i=1}^{N}\left\{\lambda_{1 i} \log \left(1-\exp \left(\exp \left(z_{i}\right)\right)\right)+\lambda_{2 i} \log \left[\exp \left(-\exp \left(v_{i}\right)\right)-\exp \left(\exp \left(z_{i}\right)\right)\right]\right. \\
& \left.\quad+\lambda_{3 i}\left(-\exp \left(v_{i}\right)\right)\right\} \\
= & \sum_{i=1}^{N}\left\{\lambda_{1 i} \log \left(1-S\left(z_{i}\right)+\lambda_{2 i} \log \left[S\left(v_{i}-S\left(z_{i}\right)\right)\right]+\lambda_{3 i}\left(-\exp \left(v_{i}\right)\right)\right)\right\}
\end{aligned}
$$


The score equations are:

$$
\begin{aligned}
& \frac{\partial \log L}{\partial \beta_{P H}}=\sum_{i=1}^{N}\left\{\lambda_{1 i} \frac{\sigma f\left(z_{i}\right)}{1-S\left(z_{i}\right)}\left(\frac{\partial z_{i}}{\beta_{P H}}\right)+\lambda_{2 i} \frac{\left[\sigma f\left(z_{i}\right)\left(\frac{\sigma z_{i}}{\partial \beta_{P H}}\right)-\sigma f\left(v_{i}\right)\left(\frac{\sigma v_{i}}{\partial \beta_{P H}}\right)\right]}{S\left(v_{i}\right)-S\left(z_{i}\right)}\right\} \\
& +\lambda_{3 i}\left(-\exp \left(v_{i}\right)\left(\frac{\sigma v_{i}}{\partial \beta_{P H}}\right)\right) \\
& =\sum_{i=1}^{N}\left\{\lambda_{1 i} \frac{\sigma f\left(z_{i}\right)}{1-S\left(z_{i}\right)}+\lambda_{2 i} \sigma \frac{f\left(z_{i}\right)-f\left(v_{i}\right)}{S\left(v_{i}\right)-S\left(z_{i}\right)}-\lambda_{3 i} \exp \left(v_{i}\right)\right\}\left(x_{i}\right) \\
& \frac{\partial \log L}{\partial \Delta}=\sum_{i=1}^{N}\left\{\lambda_{1 i} \frac{\sigma f\left(z_{i}\right)}{1-S\left(z_{i}\right)}\left(\frac{\partial z_{i}}{\partial \sigma}\right)+\lambda_{2 i} \frac{\sigma f\left(z_{i}\right)\left(\frac{\partial z_{i}}{\partial \sigma}\right)-\sigma f\left(v_{i}\right)\left(\frac{\partial v_{i}}{\partial \sigma}\right)}{S\left(v_{i}\right)-S\left(z_{i}\right)}\right. \\
& \left.+\lambda_{3 i}\left(-\exp \left(v_{i}\right)\left(\frac{\partial v_{i}}{\partial \sigma}\right)\right)\right\} \\
& =\sum_{i=1}^{N}\left\{\lambda_{1 i} \frac{\sigma f\left(z_{i}\right)}{1-S\left(z_{i}\right)}+\lambda_{2 i} \sigma \frac{f\left(z_{i}\right)-f\left(v_{i}\right)}{S\left(v_{i}\right)-S\left(z_{i}\right)}-\lambda_{3 i} \exp \left(v_{i}\right)\right\}\left(-\frac{1}{\sigma}\right) \\
& =\sum_{i=1}^{N}\left\{-\lambda_{1 i} \frac{f\left(z_{i}\right)}{1-S\left(z_{i}\right)}-\lambda_{2 i} \frac{f\left(z_{i}\right)-f\left(v_{i}\right)}{S\left(v_{i}\right)-S\left(z_{i}\right)}+\lambda_{3 i} \frac{\exp \left(v_{i}\right)}{\sigma}\right\} \\
& \frac{\partial \log L}{\partial \sigma}=\sum_{i=1}^{N}\left\{\lambda_{1 i} \frac{\sigma f\left(z_{i}\right)}{1-S\left(z_{i}\right)}\left(\frac{\partial z_{i}}{\partial \sigma}\right)\right. \\
& \left.+\lambda_{2 i} \frac{\sigma f\left(z_{i}\right)\left(\frac{\partial z_{i}}{\partial \sigma}\right)-\sigma f\left(z_{i}\right)\left(\frac{\partial z_{i}}{\partial \sigma}\right)}{S\left(v_{i}\right)-S\left(z_{i}\right)}+\lambda_{3 i}\left(-\exp \left(v_{i}\right)\right)\left(\frac{\partial v_{i}}{\partial \sigma}\right)\right\} \\
& =\sum_{i=1}^{N}\left\{\lambda_{1 i} \frac{\left(u_{i}-\Delta-x_{i}^{\prime} \beta_{A F T}\right) f\left(z_{i}\right)}{\sigma\left(1-S\left(z_{i}\right)\right)}\right. \\
& -\lambda_{2 i} \frac{\left(u_{i}-\Delta-x_{i}^{\prime} \beta_{A F T}\right) f\left(z_{i}\right)-\left(l_{i}-\Delta-x_{i}^{\prime} \beta_{A F T}\right) f\left(v_{i}\right)}{\sigma\left(S\left(v_{i}\right)-S\left(z_{i}\right)\right)} \\
& \left.+\lambda_{3 i} \frac{\left(l_{i}-\Delta-x_{i}^{\prime} \beta_{A F T}\right) \exp \left(v_{i}\right)}{\sigma^{2}}\right\}
\end{aligned}
$$




\section{Appendix 2}

\section{Derivation of $A_{11}, A_{12}$ and $A_{21}$}

Derivations of the upper left and right elements of $\mathrm{A}(\beta, \theta), A_{11}$ and $A_{12}$, are as follows:

$$
\begin{aligned}
& A_{11}^{n}(\beta, \theta)=-\frac{1}{n} \frac{\partial S_{\beta}(\beta, \theta)}{\partial \beta} \\
&=-\frac{1}{n} \sum_{i=1}^{n} \sum_{q=1}^{Q}\left[S_{i q}^{P H}\left(\frac{\partial \omega_{i q}}{\partial \beta}\right)^{\prime}+\omega_{i q}\left(\frac{\partial S_{i q}^{P H}}{\partial \beta}\right)\right] \\
&=-\frac{1}{n} \sum_{i=1}^{n} \sum_{q=1}^{Q}\left[S _ { i q } ^ { P H } \left\{\frac{S\left(t_{i(q-1)}\right)-S\left(t_{i q}\right)}{S\left(l_{i}\right)-S\left(u_{i}\right)} \frac{\left[\frac{\partial}{\partial \beta}\left(S\left(t_{i(q-1)}\right)-S\left(t_{i q}\right)\right)\right]^{\prime}}{S\left(t_{i(q-1)}\right)-S\left(t_{i q}\right)}\right.\right. \\
&\left.\left.-\omega_{i q} \frac{\left[\frac{\partial}{\partial \beta}\left(S\left(l_{i}\right)-S\left(u_{i}\right)\right)\right]^{\prime}}{S\left(l_{i}\right)-S\left(u_{i}\right)}\right\}+\omega_{i q}\left(\frac{\partial S_{i q}^{P H}}{\partial \beta}\right)\right] \\
&=-\frac{1}{n} \sum_{i=1}^{n} \sum_{q=1}^{Q}\left[S_{i q}^{P H}\left(\omega_{i q}\left(U_{i \beta}^{q}\right)^{\prime}-\omega_{i q}\left(U_{i \beta}^{o}\right)^{\prime}+\omega_{i q}\left(\frac{\partial S_{i q}^{P H}}{\partial \beta}\right)\right]\right. \\
&=-\left\{\frac{1}{n} \sum_{i=1}^{n} \sum_{q=1}^{Q} \omega_{i q} S_{i q}^{P H}\left[\left(U_{i \beta}^{q}\right)^{\prime}-\left(U_{i \beta}^{o}\right)^{\prime}\right]\right\}+I^{P H}
\end{aligned}
$$

The $p \times 1$ vector $S_{i q}^{P H}$ is the individual contribution from the $q^{t h}$ sub-interval of the $i^{\text {th }}$ subject to the partial derivative of the log likelihood with respect to $\beta$ under the $\mathrm{PH}$ model and its estimate is defined in (4). The $(p \times 1)$ vector $U_{i \beta}^{q}$ is the score of the parametric interval-censored Weibull survival model with respect to $\beta$ of the subinterval sample with lower and upper values $t_{i(q-1)}$ and $t_{i q}$ for the $q^{\text {th }}$ interval of subject $i$. The $(p \times 1)$ vector $U_{i \beta}^{0}$ denotes the score of the parametric interval-censored survival model of the original sample with lower and upper values $l_{i}$ and $u_{i}$ for subject $i$. The $(p \times p)$ matrix $\mathrm{I}^{P H}$ denotes the information matrix from the weighted $\mathrm{Cox} \mathrm{PH}$ model. The first term of equation (5) reflects the amount of variance inflation due to estimation of the weights. Without the weighting, $A_{11}(\beta, \theta)=\mathrm{I}^{P H}$ is analogous to $A_{22}(\beta, \theta)=\mathrm{I}^{A F T}$. Similarly, 


$$
A_{12}^{n}(\beta, \theta)=-\frac{1}{n} \sum_{i=1}^{n} \sum_{q=1}^{Q} \omega_{i q} S_{i q}^{P H}\left(U_{i \beta}^{q}-U_{i \beta}^{o}\right)^{\prime}
$$

where the $2 \times 1$ vector $\boldsymbol{U}_{i \theta}^{o}$ is the score of the Weibull interval-censored survival model with respect to $\boldsymbol{\theta}$ fit on the original data with lower and upper values $l_{i}$ and $u_{i}$ for subject $i$.

The lower left element of $\boldsymbol{A}(\boldsymbol{\beta}, \boldsymbol{\theta}), \boldsymbol{A}_{\mathbf{2 1}}$, consists of the derivatives of the intervalcensored Weibull score equations for $\boldsymbol{\theta}$ with respect to $\boldsymbol{\beta}_{\boldsymbol{P H}}$. Derivation of $\boldsymbol{A}_{\mathbf{2 1}}$ is as follows:

where

$$
\begin{aligned}
A_{21}^{n}(\beta, \theta) & =-\frac{1}{n} \frac{\partial U_{\theta}^{o}(\beta, \theta)}{\partial \beta} \\
& =-\frac{1}{n} \sum_{i=1}^{n} \frac{\frac{\partial U_{i \Delta}^{o}(\beta, \theta)}{\partial \beta_{P H}}}{\frac{\partial U_{i \sigma}^{o}(\beta, \theta)}{\partial \beta_{P H}}}
\end{aligned}
$$

$$
\begin{aligned}
\frac{\partial U_{i \Delta}^{o}(\beta, \theta)}{\partial \beta_{P H}}= & -x_{i}\left\{\lambda_{1 i}\left[\frac{f\left(z_{i}\right)\left(1-e^{z_{i}}\right)}{1-S\left(z_{i}\right)}-\frac{\sigma f\left(z_{i}\right)^{2}}{\left(1-S\left(z_{i}\right)\right)^{2}}\right]\right. \\
& -\lambda_{2 i}\left[\frac{f\left(v_{i}\right)\left(1-e^{v_{i}}\right)-f\left(z_{i}\right)\left(1-e^{z_{i}}\right)}{S\left(v_{i}\right)-S\left(z_{i}\right)}+\sigma\left[\frac{f\left(v_{i}\right)-f\left(z_{i}\right)}{S\left(v_{i}\right)-S\left(z_{i}\right)}\right]^{2}\right] \\
& \left.-\lambda_{3 i} \frac{f\left(v_{i}\right)}{S\left(v_{i}\right)}\right\}
\end{aligned}
$$

and

$$
\begin{aligned}
\frac{\partial U_{i \sigma}^{o}(\beta, \theta)}{\partial \beta_{P H}}= & -x_{i}\left\{\lambda_{1 i}\left[\frac{f\left(z_{i}\right)\left(1+z_{i}\left(1-e^{z_{i}}\right)\right)}{1-S\left(z_{i}\right)}-\frac{z_{i} \sigma f\left(z_{i}\right)^{2}}{\left(1-S\left(z_{i}\right)\right)^{2}}\right]\right. \\
& -\lambda_{2 i}\left[\frac{f\left(v_{i}\right)\left(1+v_{i}\left(1-e^{v_{i}}\right)\right)-f\left(z_{i}\right)\left(1+z_{i}\left(1-e^{z_{i}}\right)\right)}{S\left(v_{i}\right)-S\left(z_{i}\right)}\right. \\
& \left.\left.+\sigma \frac{\left(f\left(v_{i}\right)-f\left(z_{i}\right)\right)\left(v_{i} f\left(v_{i}\right)-z_{i} f\left(z_{i}\right)\right)}{\left(S\left(v_{i}\right)-S\left(z_{i}\right)\right)^{2}}\right]-\lambda_{3 i}\left[\frac{f\left(v_{i}\right)}{S\left(v_{i}\right)}\left(1+v_{i}\right)\right]\right\}
\end{aligned}
$$

To estimate $A(\beta, \theta)$, once can replace $\beta$ and $\theta$ with $\hat{\beta}$ and $\hat{\theta}$ after the parameter estimation converges. 
